\title{
Features of Estimation of Structural Reliability of Power Supply Systems
}

\author{
Boris Papkov ${ }^{1 *}$ and Vladimir Osokin ${ }^{1}$ \\ ${ }^{1}$ Nizhny Novgorod State Engineering and Economic University, Chair of Electrification and Automation, 606340 Knyaginino, \\ Oktyabrskaya str. h .22A, Russia
}

\begin{abstract}
Development and operation of modern power supply systems of consumers which are the part of distributed generation (DG) systems with their communication with electric power system (EPS) and renewable energy sources (RES) demand changes in estimation of their reliability indicators on the basis of corresponding mathematical apparatus. It is shown that DG systems refer to structures with several overlapping zones of action, which determines their peculiarities, which are represented by some integral characteristic - efficiency of operation, which characterizes the degree of expediency of use of the DG system in different modes. The approach based on evaluation of effectiveness of such systems is offered. Examples of calculation of relatively simple structures of DG systems performing several tasks simultaneously are given.
\end{abstract}

Keywords: reliability, structure, efficiency, distributed generation, mode

\section{Status of issue}

A specific feature of distributed generation (DG) systems is the complexity of their energy, technological and organizational structure. On the one hand, it allows to solve a complex of problems of ensuring reliable and effective power supply of consumers, and, on the other hand, to provide stable operation in case of violation of the working capacity of separate generating installations (GI) and even their groups. It is known that for such systems there is no generally accepted concept of "failure", because changes in the structure of DG system due to failures of separate GI and their connections lead, as a rule, only to decrease in its functioning since the boundaries between serviceable and faulty, as well as between operational, partially operational and non-operational states are blurred and often conditional [1]. This is due to some redundancy of the structure, presence of reserve GI (overlapping zones), switching capabilities and connections, peculiarities of relay protection (RP) and emergency automation (EA), means of correction modes (reactive power compensation and voltage regulation), possible errors of operational personnel.

Since DG systems are represented by structures with several overlapping zones of action, this determines the features of their work, which can be assessed by some integral characteristic - efficiency of functioning - the degree of expediency of using the DG system in different modes. However, issues related to the peculiarities of calculating the efficiency of systems with overlapping zones have not been fully investigated [2-5, 8, 9], which makes the topic relevant and the practical implementation of the overlapping zones method necessary.
Even formally in the state of full operability the DG system may not perform all the functions required of it. This is due to unfavorable circumstances (uneven distribution of power by renewable energy sources (RES), overload with increased consumer demand, unauthorized external influences (cyber attacks)) [7].

It should be taken into account that the behavior of autonomously operating GI of DG systems differs significantly from their behavior in the system. In the latter case, the features of hierarchical structure, modes and switching capabilities of network may appear; interdependence of failures of individual elements of DG system; interdependence (sequence) of their recoveries, etc. This leads to the need to take into account the fact that the behavior of single $K$ element of $x_{k}$ is determined by the background of some or even all other elements [4]:

$$
x_{k}(t+d t)=f_{k}\left[x_{k}(0, t), x_{1}(0, t), \ldots, x_{n}(0, t)\right],
$$

where $f_{k}$ - function, determined by the specific features of the system.

Therefore, the state of the system is determined by the $n$-dimensional random value

$$
X(t)=\left[x_{1}(t), x_{2}(t), \ldots, x_{n}(t)\right],
$$

and the trajectory of its behavior in the time interval $(t, t+\theta) n$-dimensional random function

$$
X(t, \theta)=\left[x_{1}(t, \theta), x_{2}(t \theta), \ldots, x_{n}(t \theta)\right] .
$$

Therefore, there is a need to expand the concept of "reliability" [7] and the transition to performance indicators of DG system when it performs certain tasks in specific conditions [2-5]. Efficiency indicator is a measure that quantifies the quality of functions performed by DG system, that is, a measure of usefulness of DG system operation in a certain situation while providing power

\footnotetext{
Corresponding author: boris.papkov@gmail.com
} 
supply to consumers in specific operating modes of the GI.

\section{DG system with overlapping zones of action}

Since any electric power system (EPS), including DG, belongs to the class of large systems with several levels of functioning, has a complex structure, multifunctionality, redundancy, it cannot be represented by single classical reliability indicators. Failures of its individual elements (in this case the GI and the links between them) do not lead, as a rule, to the failure of the system, but only reduce the efficiency of its functioning.

In the simplest case [8] efficiency is the probability of performing an autonomous GI task for uninterrupted power supply to consumers. The efficiency coefficient $E$ in this case additionally acquires the meaning of probability that the implementation of this task will not be disrupted due to the impossibility of implementing some modes (failures). We consider that $N_{\Sigma}$ is a set of all possible operating modes of GI implemented to provide power supply to consumers; $N$ is a set of actually existing modes. However, some additional permissible modes are possible, forming a plurality of $N_{0}$ together with $N$. Then $N \in N_{0} \in N_{\Sigma}$ (fig. 1).

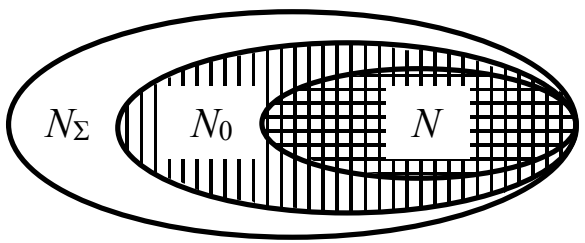

Fig. 1. Possible ratio of GI modes.

Efficiency here is the probability $p$ that the mode $n$ belongs to the set $N$, that is, $E=p\{n \in N\}$. Similarly is $E_{0}=p\left\{n \in N_{0}\right\}$. The probability that the mode of operation of GI simultaneously belongs to two sets $N$ and $N_{0}$ will be

$$
p\left\{n \in N, n \in N_{0}\right\}=p\{n \in N\},
$$

because $N \subset N_{0}$ (fig. 1).

According to the probability multiplication theorem we get

$$
p\left\{n \in N, n \in N_{0}\right\}=p\left\{n \in N_{0}\right\} \cdot p\left\{n \in N \mid n \in N_{0}\right\} .
$$

The efficiency coefficient is defined as

$$
\begin{aligned}
E=\frac{p\{n \in N\}}{p\left\{n \in N_{0}\right\}}= & \frac{p\left\{n \in N, n \in N_{0}\right\}}{p\left\{n \in N_{0}\right\}} \\
& =p\left\{n \in N \mid n \in N_{0}\right\}
\end{aligned}
$$

The given expression (1) has probability that power supply to consumers will be carried out in any mode of GI operation.

Considering the difficulty of obtaining estimates of efficiency of DG systems, it is necessary to develop and improve the corresponding mathematical apparatus. In general, the efficiency of the DG $E_{\text {syst }}(t)$ is come down to calculation according to the formula offered in [2]

$$
E_{\text {syst }}(t)=\sum_{k=1}^{2^{n}} p_{X_{k}}(t) E_{X_{k}},
$$

where $X=\left(x_{1}, x_{2}, \ldots, x_{n}\right)$ is system condition of DG; $n$ is number of system's elements, being in two conditions ( $x_{i}=1$ - efficient, $x_{i}=0$ - failure); $p_{X_{k}}(t)-$ probability that the system is $X_{k}$ condition at time $t ; E_{X_{k}}$ - efficiency of system being in $X_{k}$ condition.

When $n$ is large, the calculation of efficiency according to (2) is quite complicated. Therefore, we consider the approach offered in $[2,3,5]$. It is based on the fact that in some territory there are several GI united in the system, providing power supply to consumers not only directly connected to "their" GI, but also (with a sufficient reserve of power and network capacity) other, geographically close and located in the overlapping zones of adjacent GI.

If in the system of $n$ elements the $i$ element with probability $p_{i}$ is in good condition, the results of its operation apply to the area $S_{i}$. Coverage zones of two, three, etc. elements can intersect, forming area $S_{i, j}, S_{i, j, k}, \ldots, S_{i, j, k, \ldots, n}$ with corresponding elements of influence. In general case such zones can be $2^{n}$. Scope of the whole system is represented by the union of all zones of action of elements $S=\bigcup_{i=1}^{n} S_{i}$ [2].

Suppose that conditional power district representing system of DG and consisting of four GI (power plants), each of which provides operation of consumers of electric power of "their" zone. $\Omega=\bigcup_{i=1}^{4} Z_{i}$. There are areas in each zone, which intersect adjacent two or more zones. Consequently, system with overlapping zones of action is formed.

The part of zone that does not intersect with others is designated as $S_{i}, \quad(i=\overline{1,4})$, and the areas of intersection are $S_{i, j}, S_{i, j, k}(i=j=k=\overline{1,4})$. All selected zones do not intersect and define the district $\mathrm{S}$ of GI operation of investigated DG system (Fig. 2).

The efficiency coefficients of selected areas are denoted by $W$ with corresponding indexes. If, for example, GI whose main consumers are located in zone $Z_{2}$ fails, the efficiency coefficients of areas covered by this zone will change. Instead of $E_{12}, E_{23}, E_{24}, E_{234}$ we get $E_{1}, E_{3}$, $E_{4}, E_{34}$. Naturally, in each practical problem the efficiency coefficients for intersection areas are determined individually.

Such division of energy district of DG system, consisting of $n$ GI can significantly reduce the number of selected areas relative to the value of $2^{n}$, that is, simplifies the calculation of the efficiency coefficient of DG system.

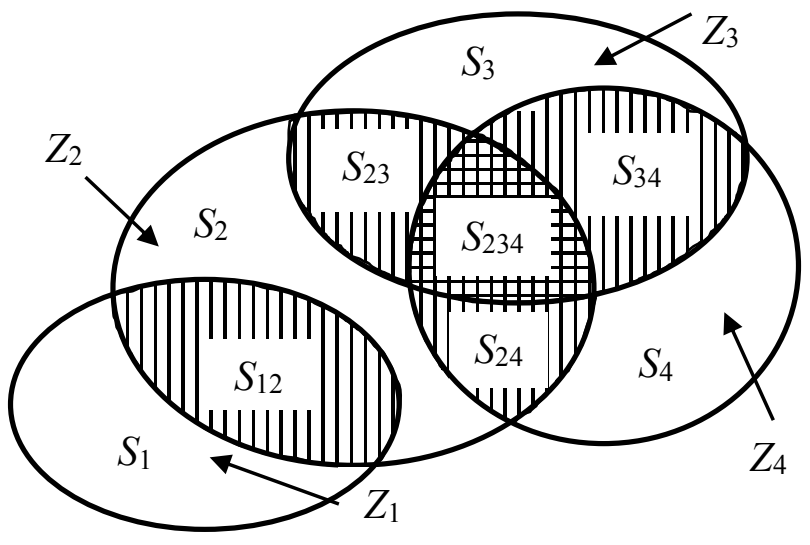

Fig. 2. Zones of operation of GI of DG. 
Its calculation includes calculation of efficiency coefficients of each areas and their summation taking into account

- power output from generating units;

- switching capabilities of power supply schemes;

- network mode settings;

- composition, features of operation and installations of RP and EA systems;

- power consumption and technological features of consumers located in corresponding zone;

- other indicators characterizing the transition of DG system to restrictive regime.

The component of efficiency coefficient $e_{i}$ in resulting sum for $S_{i}$ area without $S$ overlapping with other zones is defined as

$$
e_{i}=S_{i} E_{i} p_{i}
$$

where $p_{i}$ is probability of GI failure in $Z_{i}$ area with power supply failure of consumers in $S_{i}$. zone.

The component for the overlap area of $Z_{i}$ and $Z_{j}$ zones is equal to

$$
e_{i j}=S_{i j}\left(E_{i j} p_{i} p_{j}+E_{i} p_{i} q_{j}+E_{j} q_{i} p_{j}\right)
$$

The component formed by the overlap of three zones $Z_{i}, Z_{j}$ and $Z_{k}$

$$
\begin{aligned}
e_{i j k}= & S_{i j k}\left(E_{i j k} p_{i} p_{j} p_{k}+E_{i j} p_{i} p_{j} q_{k}+E_{i k} p_{i} q_{j} p_{k}\right. \\
& +E_{j k} q_{i} p_{j} p_{k}+ \\
& \left.+E_{i} p_{i} q_{j} q_{k}+E_{j} q_{i} p_{j} q_{k}+E_{k} q_{i} q_{j} p_{k}\right) .
\end{aligned}
$$

Estimation of efficiency coefficients of each area with more zones is done in the same way.

Note that efficiency coefficient determines the average level of quality of the system, due to the reliability of its elements. It can be dimensionless (probability of execution of requirements of consumer) and have the dimension (the amount of generated power, the amount of consumption, profit, damage, etc.) In case of GI failure in $Z_{i}$ zone losses (damage) are determined by consequences of power supply failure of consumers located in $S_{i}$ area. In other areas of overlap with other zones, there will (or may) be some decrease in the efficiency of functioning of consumers (reduced productivity, changes in the parameters of the technological process and (or) the quality of products) located in these zones [1]. The damage $Y_{i}$ in zone $S_{i}$ can be estimated by expression

$$
Y_{i}=S_{i} E_{i}+S_{i j}\left(E_{i j}-E_{i}\right)+S_{i j k}\left(E_{i j k}-E_{i}\right)+\ldots \text {. }
$$

An approximate estimate of the magnitude of total damage $Y_{\Sigma}$ based on the probability $p_{i}$ of failure GI in different zones of DG systems will be

$$
Y_{\Sigma}=\sum_{i=1}^{n} p_{i} Y_{i} \text {. }
$$

\section{Bases of estimation of efficiency of DG systems with overlapping zones of action}

Consider a system of DG consisting of two identical GI, each of which can operate in two modes: $x_{1}$ и $x_{2}$.

If both modes $x_{1} \cup x_{2}$ are possible then the effectiveness of system of DG $-E=S_{2}$; if $\bar{x}_{1} \cup x_{2}$ or $x_{1} \cup \bar{x}_{2}$, then $E=S_{1}$, (if $\bar{x}_{i}, i=1,2$ mode fails) and $S_{1}<S_{2}$; if $\bar{x}_{1} \cup \bar{x}_{2}$, then $E=0$ [9].
The state of DG system is characterized by vector with components

$$
\left\{x_{1}^{(1)} x_{2}^{(1)} x_{1}^{(2)} x_{2}^{(2)}\right\}
$$

where the upper index is the number GI, lower index - corresponding mode.

Each component (3) takes two values: $x_{i}^{j} \mathrm{j}$ - power supply mode of consumers is performed; $\bar{x}_{i}^{j}$ - the mode is impracticable.

When $E=0$ we have:

$$
\left\{\bar{x}_{1}^{(1)} \bar{x}_{2}^{(1)} \bar{x}_{1}^{(2)} \bar{x}_{2}^{(2)}\right\} \text {. }
$$

When $E=S_{1}$ two modes can be: $a$ ) arbitrary, but only one component of vector (3) takes the value $x_{i}^{j}$, and other $\left.-\bar{x}_{i}^{j} ; b\right)$ DG system state is characterized by one of vectors

$$
\left\{\bar{x}_{1}^{(1)} x_{2}^{(1)} \bar{x}_{1}^{(2)} x_{2}^{(2)}\right\},\left\{x_{1}^{(1)} \bar{x}_{2}^{(1)} x_{1}^{(2)} \bar{x}_{2}^{(2)}\right\} .
$$

When $E=S_{2}$ two modes are also possible: $a$ ) at least three components of vector (3) take the value $x_{i}^{j}$ (one of GI is completely serviceable); b) DG system state is characterized by one of vectors

$$
\left\{\bar{x}_{1}^{(1)} x_{2}^{(1)} x_{1}^{(2)} \bar{x}_{2}^{(2)}\right\},\left\{x_{1}^{(1)} \bar{x}_{2}^{(1)} \bar{x}_{1}^{(2)} x_{2}^{(2)}\right\} \text {. }
$$

Comparison of expressions (4) and (5) shows that in both cases the system consists of two GI operating in one of possible modes; the number of impossible modes is also the same, but efficiency indicators are different. The difference is determined by the nature of conditions for power supply of consumers. According to (4) the DG system is fully operational at the expense of two partially operational GI.

First of all the estimation of effectiveness of DG systems should be based on fundamental knowledge about their structural, technological and organizational peculiarities, principles of operation of the main and control elements, relay protection systems and automation, which are part of them $[10,11]$. The order of even the preliminary estimation of the effectiveness of DG systems is determined by the following sequence:

- obtaining information on main circuit-mode characteristics and technical and economic indicators of operation of DG system;

- possible tasks of DG system operation are investigated;

- DG system operation conditions are determined in parallel with EPS, independently and in combined mode;

- estimated the expected frequency of repetition of tasks and modes of DG system operation;

- functional diagram of the system is drawn up;

- DG system is divided into separate elements (zones, regions);

- quantitative measure of functioning quality of acceptable for this system of DG is chosen;

- reliability indicators of elements characterizing probability of state of each element at different moments of time are calculated; 
- probabilities of average (if necessary permissible and/or limit) states of DG system are calculated on the basis of probabilities of states of separate elements;

- efficiency indicators of possible states of DG system are evaluated.

If for each state of DG system it is possible to estimate the "instant" value of its functioning efficiency (output effect), the efficiency coefficient is defined as the average value of the output effect for all states of the system [2].

\section{Examples of indicative estimation of the effectiveness of DG systems}

We illustrate the method of calculating the efficiency coefficient of DG system on simple examples in which the traditional calculation of reliability indicators is quite complex.

1). Non-intersecting coverage zones of $G I$ in $D G$ system. Each of two identical GI in DG system provides power supply to consumers in its zone with probabilityp $=0,9$. The coefficient of readiness of each GI will take as $K_{g}=0,95$. The coefficient of interval readiness $K_{g}^{*}\left(t, t+t_{0}\right)$ is introduced in order that the system was efficient not only in any required timepoint, but also it would be able to work smoothly during the required time interval $t+t_{0}$. In general case $p^{*}(t) \neq p$ is because $p$ is probability of no-failure operation, since condition of full working capacity, and $p^{*}(t)$ is probability of nofailure operation, since one of possible intermediate conditions of working capacity. For simplicity, assume that $t \rightarrow \infty$ and $p^{*}(t)=p=0,9$. Then

$$
K_{g}^{*}(t)=K_{g} p^{*}(t)
$$

In case of failure of one of GI DG system splits into two independent subsystems, each of which can provide only half the load of consumers. The efficiency coefficient of one GI is defined as

$$
e_{g i}(t)=0,5 K_{g} p=0,5 \cdot 0,95 \cdot 0,9=0,4275 \text {. }
$$

Therefore, the considered system of DG provides the efficiency coefficient equal to $E_{\Sigma}=2 e_{g i}=0,855$.

2). Point-by-point estimation of operation efficiency of DG system in case of the simplest GI. The power supply system (DG) consists of two identical power plants (GI): $S a$ and $S b$, providing power to consumers with the maximum power consumption $P_{\Sigma}=36 \mathrm{MW}$. The $S a$ station operates in the power output mode in the range from $P=0$ to $P=22 \mathrm{MW}$, and the $S b$ station from $P=14$ to $P=36 \mathrm{MW}$. The power distribution zones of these stations are shown in Fig. 3.

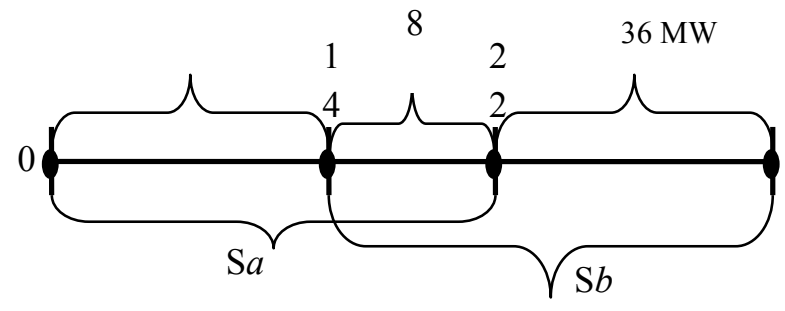

Fig. 3. Zones of power distribution of GI.
It is required to find the probability of providing the load of consumers appearing at any time in the range $0 \leq P \leq 36 \mathrm{MW}$, if the reliability of providing the necessary power by these stations at this moment: $R_{a}=$ $R_{b}=0,95$. Thus probability of reliable power supply of the consumers which are in a zone of action of each of GI $-R_{1}=R_{2}=0,9$, and in zone of simultaneous action of both stations (in a zone of overlapping)

$$
\mathcal{P}=1-\left(1-R_{1}\right)^{2}=0,99 \text {. }
$$

Suppose that the load of consumers at given time is $P_{0} \mathrm{MW}$. The probability that the DG system will be able to provide it at this point in time

$$
\mathcal{P}_{0}=R_{a} \cdot R_{b}=0,95 \cdot 0,95 \approx 0,9 \text {. }
$$

At the interval from $P=14$ to $P=22 \mathrm{MW}$ (overlap zone $-P_{\Pi}=8 \mathrm{MW}$ ), the load of the consumer is provided by both stations, and within zones (from $P=0$ to $P=14$ and from $P=22$ to $P=36 \mathrm{MW}$ ) - by one GI.

The efficiency index $E_{0}$ of DG system state at given load $P_{0} \mathrm{MW}$ is determined by weighted average

$$
\begin{aligned}
E_{0}=\frac{P_{S_{a} \cap S_{b}}}{P_{\Sigma}} \mathcal{P}+ & \frac{P_{S_{a}-\left(S_{a} \cap S_{b}\right)}+P_{S_{b}-\left(S_{a} \cap S_{b}\right)}}{P_{\Sigma}} \mathcal{P}_{0} \\
& =\frac{8}{36} \cdot 0,99+\frac{14+14}{36} \cdot 0,9=0,92 .
\end{aligned}
$$

Note that "weight" here is states of DG system, and not its separate elements (GI). In the same DG system, "weight" of an element essentially depends on the state for which it is considered. If the failure of $i$-element occurred for all working elements, it is obvious that it is accompanied by consequences different from the case when this $i$-element failed after the failure of one $j$ $(i \neq j)$ or several elements of this system. However, for DG systems, in many cases, an element is more important than another in one state of the system proves to be more important in another state of the system.

The probability of system state when the load limits $0<P_{a}<22 \mathrm{MW}$ has one station $\mathrm{Sa}$, and in the range $22<P<36 \mathrm{MW}-$ none,

$$
\left.\mathcal{P}_{a}=R_{a}\left(1-R_{a}\right)=0,95 \cdot 0,05\right) \approx 0,05 .
$$

The efficiency index $E_{a}$ of DG system state at a load of $P_{a} \mathrm{MW}$ will be determined as

$$
E_{a}=\frac{22}{36} \cdot 0,9+\frac{14}{36} \cdot 0=0,55 \text {. }
$$

Since in given example the states of $S a$ and $S a$ are absolutely identical, so we get the resulting efficiency of operation of analyzed DG system:

$$
\begin{gathered}
E_{\Sigma}=\mathcal{P}_{0} E_{0}+2 \mathcal{P}_{a} E_{a}=0,9 \cdot 0,92+2 \cdot 0,05 \cdot 0,55= \\
0,883 .
\end{gathered}
$$

3). Estimation of technical efficiency of DG system at a certain time interval. The power supply system (GI) consists of two identical power plants (GI). In case of operability of both stations the load provided by them is $P$ MW. In case of failure of one of them, the value of provided load is reduced to the value of $0,3 P$. In case of failure of two stations, the power supply of consumers is disrupted completely. The failures of GI are independent events. The probability of failure-free operation of each GI obeys the exponential distribution law

$$
p(t)=e^{-\frac{t}{T}}=e^{-\lambda t} .
$$

Considering DG systems of this type it is necessary to take into account that the GI will not give the required power to consumers when stopped as a result of preven- 
tive maintenance, as well as the impact of any external factors (weather conditions, if the composition of the GI is determined by RES). However, in order to simplify the problem, we assume that the distribution law remains exponential.

If we take the duration of the calculation period $\tau=t$ equal to the average uptime of each GI $t=T=1$ in a year, the probability of uptime GI will be

$$
p=e^{-\frac{t}{T}}=e^{-1}=0,368 .
$$

Indicators of technical efficiency of DG system states are determined by multiplication of transmitted power on time of operation:

1) technical efficiency of DG system operation when both stations are operable during considered period $\tau$

$$
E_{0}=P \tau
$$

2) technical efficiency of DG system operation, when the first GI failed at the moment of $t_{i}$

$$
E_{i}\left(t_{i}\right)=0,3 P \tau+0,7 P t_{i} \quad t_{i}<\tau ; \quad i=a, b ;
$$

3) technical efficiency of DG system operation, when the first GI failed at the moment of $t_{i}$, and the second one - at the moment of $\mathrm{t}_{\mathbf{j}}$

$$
E_{i j}\left(t_{i}, t_{j}\right)=0,3 P t_{j}+0,7 P t_{i} \quad t_{i}<t_{j}<\tau,
$$

The resulting estimation of efficiency of analyzed DG system according to the theory set in $[4,5]$ is got according to formula:

$$
\begin{gathered}
E=p^{2} P \tau+2 p\{0,3 P \tau(1-p)+0,7 P \tau[1-p(1+\lambda \tau)]\} \\
\left.+\quad p(1+\lambda \tau)+0,25 p^{2}(1+2 \lambda \tau)\right]+ \\
+2\left\{0,3 P \tau\left[0,75-p+(0,75+0,5 \lambda \tau) p^{2}\right]\right\}= \\
+0,7 P \tau[0,25-p+0,368\{0,3 P \tau(1-0,368) \\
\left.=0,368^{2} P \tau+2 \cdot 0,7 P \tau[1-0,368(1+1)]\right\}+ \\
+2\{0,3 P \tau[0,75-0,368(1+1)+0,25 \\
\left.\cdot 0,368^{2}(1+2)\right]+ \\
\left.+0,7 P \tau\left[0,25-0,368+(0,75+0,5) 0,368^{2}\right]\right\}= \\
=(0,135+0,28+0,138) P \tau=0,553 P \tau
\end{gathered}
$$

The analyzed DG system can be considered as a system with additive efficiency indicators $[4,5]$, since each GI of this system contributes its independent share of $c_{k}$ to the overall output effect. Then the effectiveness of system will be determined according to following expressions

$$
\begin{gathered}
E_{0}=P \tau \mathcal{P}_{0}=P \tau\left[1-(1-p)^{2}\right]=P \tau[1- \\
\left.(1-0,368)^{2}\right]=0,6 P \tau ; \\
E_{i}\left(t_{i}\right)=0,3 P \tau \mathcal{P}_{0}+0,7 P t_{i}\left(1-e^{-\frac{t_{i}}{\tau}}\right) ; \\
E_{i j}\left(t_{i}, t_{j}\right)=0,3 P t_{j}\left(1-e^{-\frac{t_{j}}{\tau}}\right)+0,7 P t_{i}\left(1-e^{-\frac{t_{i}}{\tau}}\right) ; \\
E(t)=\sum_{k=1}^{n} E_{k} c_{k}=E_{0} c_{0}+E_{i}\left(t_{i}\right) c_{i} \\
+E_{i, j}\left(t_{i, j}\right) c_{i, j}, \quad \sum_{k=1}^{n} c_{k}=1,
\end{gathered}
$$

where $c_{k}$ is the coefficient of ratio participation of each GI operating in DG system.

\section{Conclusion}

Despite the fact that the reliability of the elements is increasing, the rate of this growth lags behind the rate of increase in the complexity of the system. The nature of functions performed by DG system requires its high reliability, but a large number of elements, their links and modes of operation can adversely affect the efficiency of the system as a whole.

The absence of practical methods for estimation of effectiveness of DG systems, characterized by a variable structure that can be changed at random and intersecting GI zones of operation, is extremely relevant and requires solving these problems, meeting the requirements of competitiveness in the world market.

The effectiveness of operation of DG system must be assessed by a specialist familiar with the system as a whole, who knows the requirements for it, its structure, modes, methods and means of control and protection.

\section{References}

1. B.V. Papkov, A.L. Kulikov Theory Systems and System Analysis for electric power engineers (Publishing house Urait, 2016)

2. I.A. Ushakov Course of the theory of reliability of systems (Dropha, 2008.)

3. K. Rainshke, I.A. Ushakov Reliability evaluation of systems using graphs(Radio и communication, 1988)

4. I.A. Ushakov Effi. of fun. of com. Sys. About reliability of complex technical systems (Soviet radio, 1966)

5. I.A. Ushakov Research methods of efficiency of functioning of technical systems (Publ.house Zhnanie, 1976)

6. A.L. Kulikov, V.L. Osokin, B.V. Papkov Prob. and pec. of dist. elec., NGIEI, 11 (90) (2018)

7. B.V. Papkov Vuln. and resil. of elec. facil. Research and ensuring the reliability of energy systems, 68 (Irkutsk: ISEM SO RAN, 2017)

8. E.V. Dzirkal Specifying and verifying reliability requirements for complex products (Radio и communication, 1981)

9. V.V. Plyamovatyi To the theor of "surv" of comp. syst. Main Issues of Theory and Practice of Reliability (Soviet radio, 1980)

10. Yu.E. Gurevich, P.V. Ilyushin Features of calculations of modes in energy district with distributed generation Nizhny Novgorod (Nizhny Novgorod Institute of Management, Branch of Ranepa, 2018)

11. P.V. Ilyushin, A.L. Kulikov Automatic control of normal and emergency modes of energy districts with distributed generation (Nizhny Novgorod Institute of Management, Branch of Ranepa, 2019) 